\title{
A BROAD SURVEY ON FEATURE EXTRACTION METHODS FOR FINGERPRINT IMAGE ANALYSIS
}

\author{
Ramesh Chandra Sahoo \\ Research Scholar, Utkal Unuversity, Bhubaneswar, India \\ Sateesh Kumar Pradhan \\ Deaprtment of Computer Science, Utkal Unuversity, Bhubaneswar, India
}

\begin{abstract}
This paper focused on the study of various feature extraction techniques applied for fingerprint identification, verification and classification as it is the most important step for image processing. Feature extraction techniques are classified into local (low level) and global (high level) features. Global features such as arch, loop, delta and whorl where as local features such as ridge end and bifurcation called minutiae are majors of automatic fingerprint recognition system. In this study, it has been observed that most of the fingerprint recognition systems are based on minutiae features. In this paper we analyze the various feature extraction methods used so far with their mathematical background to the readers.
\end{abstract}

Keywords: Feature extraction, Fingerprint.

Cite this Article: Ramesh Chandra Sahoo and Sateesh Kumar Pradhan, A Broad Survey on Feature Extraction Methods for Fingerprint Image Analysis, International Journal of Computer Engineering and Technology, 10(2), 2019, pp. 14-24.

http://iaeme.com/Home/issue/IJCET?Volume=10\&Issue=2

\section{INTRODUCTION}

Fingerprints have been used as a most reliable personal authentication system in today's digital world as because of stability through life and uniqueness (i.e the set of fingerprints of two persons are not same). A fingerprint pattern consists of ridges and valleys. Fingerprint recognition systems are broadly divided into three steps as (1) pre-processing, (2) extracting relevant features and (3) Pattern identification, or recognition or classification. Feature extraction is the process of reducing a higher dimensionality feature space to a lower dimensionality features to get most relevant information (less data more information) from a pattern. Instead of feeding the whole pattern having much data, but not much information to the further training or matching step, most relevant information should be taken into consideration to reduce the time and space of an algorithm. Feature extraction step basically carried out after pre-processing step where the image pattern was refined and the quality of 
image should improved. Feature selection and classification are two phases of feature extraction step that should be done carefully so that in the recognition or pattern classification step it provides the desired output. In case of fingerprint image the features are described in three levels as:

Level 1: Ridge orientation and frequency

Level 2: ridge skeletons

Level 3: outer and inner contours of ridges

\section{LEVEL I FEATURES}

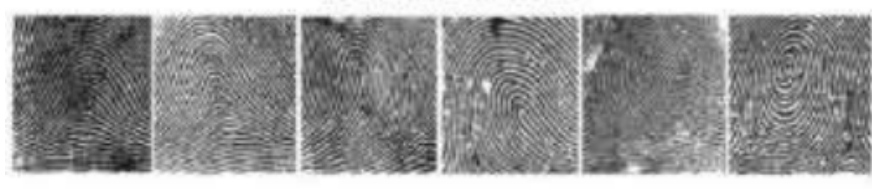

ARCH TENTEDARCH LETLOOP RGCHLLOP DOUBLELOOP WHORL
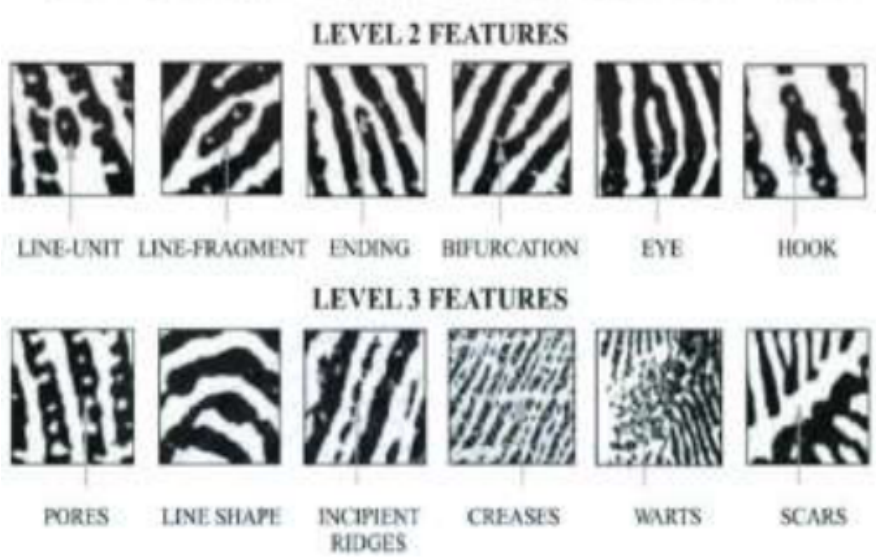

Figure 1 Fingerprint minutiae points at different levels

\section{LITERATURE REVIEW}

In the field of feature extraction techniques for fingerprint identification, verification and classification, various works have been done so far. In this paper our aim is to introduce these methods to the readers to get the updated information and their working principles till today. Enhancement of the fingerprint is carried out by the directional field, and together with for classification, singular points are used, while the minutiae features are used for matching. Many feature extraction approaches have been proposed for fingerprint pattern recognition and most of these proposed systems are based on local minutiae features. In the following section some papers we have studied are discussed.

Moayer and $\mathrm{Fu}$ [1], in this paper, they demonstrate a syntactic approach by using iterative operator (Laplacian) with a thresholding (dynamic) algorithm to binarize the fingerprint images. Verma, M.R., A.K. Majumdar, and B. Chatterjee [2], in this paper, they proposed a fuzzy approach to maintain the number of black and white pixels for each neighbourhood by using a adaptive thresholding. O'Gorman and Nickerson [3], in this paper they proposed filter bank oriented enhancement of fingerprint images with a matrix. The elements of this matrix are the tangent direction to the ridgelines of the corresponding image. It was also suggested to binarization of the image through this method. Sherlock, B.G, D.M. Monro, and K. Millard. [4], proposed a filter of frequency domain by position depended filters for enhancement and binariztion of fingerprint images. Szekely and Szekely [5], in this paper they proposed a fingerprint detection technique based on minutiae points where they computed the divergence of the directional image from binary fingerprint images. In this survey it has been observed that many automated fingerprint recognition systems were 
proposed through enhancement of fingerprint images to overcome noise in the fingerprint images and various neural network approaches were proposed to recognise and classification of fingerprint image. Mehtre, B.M., [6], proposed a analysis of noisy fingerprint images with their identification with the system. Weber. D.M [7], in this paper they proposed cost effective frequency domain filtering method for enhancement and binarization of fingerprint images and finds the minutiae by using local thresholds. Leung, M.T., W.E. Engeler, and P. Frank[8], proposed minutiae feature extraction through a multilayer perceptron after applying a bank of Gabor filters. Leung, W.F., S.H. Leung, W.H. Lau, and A. Luk[9], in this paper they proposed a method to extract the minutiae by using a three-layer perceptron from thinned binary fingerprint images. Hung. D.C.D.[10] and Xiao, Q. and H. Raafat [11], in these papers they proposed an algorithm to improve the speed and accuracy of the network from minutiae features by eliminate false minima. Jianjiang Feng and Anil K. Jain et al. [12], in this paper they proposed minutiae based fingerprint matching method that reconstructs the whole fingerprint with few false minutiae. Arun Ross, Jidnya Shah and Anil K. Jain et al. [13], in this paper they proposed $\mathrm{n}$ algorithm for reconstructing fingerprint images from minutiae points due to the orientation field of minutiae points. Anil K. Jain, Fellow, IEEE, Yi Chen , IEEE, and Meltem Demirkus, IEEE et al. [14], here they proposed a matching algorithm that uses features of level1, level2 and level3. M. Tico, E. Immonen, P. Ramo, P. Kuosmanen, and J, Saarinen[15], In this paper they proposed a Discrete Wavelet Transform on the cropped fingerprint image and calculated the standard deviations of the DWT coefficient for matching. S. Tachaphetpiboon and T. Amornraksa[16], In their implementation they applied DCT and DWT feature extraction methods for the same sub images and calculated the distribution in frequency and the standard deviations are calculated from nine overlapping regions for recognition. S.Malathi and Dr.C.Meena[17], In this paper they applied Local Binary Pattern (LBP) method. Here they extract pores of fingerprint images by taking window size of 32X32. For matching they measured the distance between two lbp feature histograms. M.P Dale, M.A Joshi, M.K Sahu[18], in this paper they applied Discrete Cosine Transform (DCT) feature extraction directly to the fingerprint images without any pre-processing by dividing into various block and calculating the standard deviations from the feature vectors for matching. C. J. Lee and S. D. Wang[19], proposed a Gabor filter feature extraction method. They used six Gabor filters, $\left(0^{\circ}, 30^{\circ}, 60^{\circ}, 90^{\circ}, 120^{\circ}, 150\right)$ anf a frequncy. $\mathrm{F}=10$ to extract the features. Nanni, Loris \& Lumini, Alessandra[20], proposed a Local Binary Pattern (LBP) feature extraction method for a hybrid fingerprint matching system by subdividing the image into sub-windows, a segmentation is performed to discard background, then each subwindow is passed through a bankmof Gabor filters and LBP and then matching is done by Euclidean distance between them. Shashi Kumar D R1, K B Raja2, R K Chhotaray3, Sabyasachi Pattanaik[21], proposed a DWT feature based fingerprint recognition system for non minutiae features. They calculated the Dominant local orientation angle $\theta$ and Coherence are computed on LL band only and compare with the stored database fingerprint on Euclidean distance method. Yongxu, Wang \& Xinyu, Ao \& Yuanfeng, Du \& Li, Yongping[22], proposed Principal Component Analysis feature extraction method to extract statistical features of fingerprint images. This feature vector is employed to segment of ROI of the fingerprint image. Chhaya Sunil Khandelwal, Ranjan Maheswarib, U. B. Shinde [23], reviewed on PCA and Fisher's LDA on both iris and fingerprint images for authontication of individual by matching these features. Yager $\mathrm{N}$, Amin $\mathrm{A}[37]$, proposed a fingerprint classification methods into five major classes, known as Henry classes. These are level-1 features of a gray scale fingerprint image namely loop (left and right), Arch, Whorl, Tented Arch. By taking these features they proposed the matching algorithm. Candela G[39], worked on orientation field information of global and local ridge patterns in fingerprint images. Chong M, Ngee T, Jun L, Gay K[40], worked on Bspline curves representation of ridges and 
these curves are used as features for further representation of other steps of matching algorithm.

\section{FEATURE EXTRACTION}

In this paper, our main aim is to present various feature extraction methods such as FFT, DWT, DCT, PCA, LBP, LPQ, Gabor Filter and their algorithms used so far to the research scholars and readers for better understanding and implementation. In any pattern recognition system, feature selection and classification are the two major steps where feature selection step is more critical since the classifier will not be able to work properly, if feature selection process was not good enough. Then these feature vectors are used in pattern recognition and classification process in further steps. In general if we say feature extraction is the process of extracting precise reduced features from a raw high features image pattern. The importance of feature extraction method is to reduce the dimensionality of an image with reliable and sufficient information for further step of image processing. In this paper we are also providing some methods for feature extraction used in fingerprint images to the readers for their better understanding and in their future research activity in this field.

\subsection{Gabor Filter Features}

Gabor filters are special types of bandpass filters basically used for texture analysis, edge detection and feature extraction in image processing. In fingerprint feature extraction when Gabor filter is applied, it gives the highest response at edges and at points where texture changes i.e on ridges and furrows. The general form of Gabor filter is given as:

$$
g(x, y, f, \theta, \varphi, \sigma, \gamma)=\exp \left(-\frac{x^{\prime 2}+\gamma^{2} y^{\prime 2}}{2 \sigma^{2}}\right) \exp \left(i\left(2 \pi \frac{x^{\prime}}{f}+\varphi\right)\right) \ldots
$$

Where:

$\sigma<-i$ s the standard deviation of the gaussian function

$\theta<-i$ s the orientation of the normal to the parallel stripes of the Gabor function

$f<-i s$ the frequency of the sinusoidal factor in the equation

$\gamma<-i$ the spatial aspect ratio

$\varphi<-i$ s the phase offset

$x^{\prime}=x \cos \theta+y \sin \theta$ and

$y^{\prime}=-x \sin \theta+y \cos \theta$

In the above equation, $\sigma$ (sigma) controls the width of the Gaussian envelop, $\theta$ (theta) controls the kind of features of the filter at various angles between 0 to 180 degree, $\gamma$ (gamma) controls the ellipticity of Gaussian function, $\omega(\mathrm{psi})$ that controls the phase offset and $\mathrm{f}$ controls the variations on frequencies.

Ksize: is the size of convolution, basically set to a square size (i.e the fingerprint image is divided into $\mathrm{nXn}$ non overlapping sub images) and then apply Gabor filter for different $\theta$ angles, frequencies and standard deviations. Then the component of the finger is the average absolute deviation of each $\mathrm{n} \mathrm{X}$ n block of the different filtered images.

\subsection{Fast Fourier Transform (FFT)}

FFT is a fast algorithm that computes the Discrete Fourier Transform (DFT) by converting the image from spatial domain to frequency domain which reduces the time complexity from $\mathrm{N}^{2}$ to $\mathrm{N} \log _{2} \mathrm{~N}$ of $\mathrm{N}$ points. And then apply inverse FFT to the image to reconvert to spatial domain. 
First decompose the gray scale fingerprint image into $\mathrm{w} \mathrm{X} \mathrm{w}$ non overlapping sub images, where $f(x, y)$ is the gray value of the fingerprint image $\mathrm{f}$ in the block $(x, y)$. Apply FFT to each sub image and calculate the pattern for each class and the result $f$ block $(p, q)$ of that block in positin $(\mathrm{p}, \mathrm{q})$ is given as:

$f \_b l o c k(p, q)=\sum_{x=0}^{w-1} \sum_{y=0}^{w-1} f(x, y) * e^{-j 2 \pi\left(\frac{x p}{w}+\frac{y q}{w}\right) .}$

Where $\mathrm{p}, \mathrm{q}=0,1,2 \ldots, \mathrm{w}-1$.

To enhance a specific block, we may multiply the FFT with the magnitude, where the magnitude of a block is given by:

$\mathrm{FFT}=\operatorname{abs}(\mathrm{F}(\mathrm{p}, \mathrm{q}))=|\mathrm{F}(\mathrm{p}, \mathrm{q})|$. And the enhanced block we get is

$h(x, y)=F^{-1}\left[F(p, q) *|F(p, q)|^{k}\right]$

and $F^{-1}(F(p, q))$ is done by:

$f(x, y)=\frac{1}{w w} \sum_{x=0}^{w-1} \sum_{y=0}^{w-1} F(p . q) * \exp \left\{j^{2 \pi} *\left(\frac{x p}{w}+\frac{y p}{w}\right)\right\}$

Where $\mathrm{x} . \mathrm{y}=0,1,2$, $\mathrm{w}-1$.

Then the direction of the local flow orientation of each block of size w X w is computed which returns ROI of the image.

\subsection{Principal Component Analysis (PCA)}

PCA is a technique used for identification of a smaller number of uncorrelated variables known as principal components from a larger set of data that still contains the most information in the large set. In fingerprint recognition system it extract meaningful features from high dimensional data in a way that helps us interpret the major trends in our data. It takes the advantage of eigenvector properties for determination of selected object orientation. The steps of PCA feature extraction are as:

- Let there are $\mathrm{N}$ input images each of size (K X K), convert each such images in a single vector of form $(K . K \times 1)$, thus the $2 \mathrm{D}$ matrix of all $N$ images be $K . K \times N$ denoted as $I_{N}$ is given as:

$$
I_{N}=\left[\begin{array}{ccc}
i_{1}^{1} & i_{1}^{2} \cdots & i_{1}^{N} \\
\vdots & \ddots & \vdots \\
i_{K * K}^{1} & i_{K * K}^{2} \cdots & i_{K * K}^{N}
\end{array}\right]
$$

Where $i_{x}^{p}$ is the $\mathrm{p}^{\text {th }}$ image's $\mathrm{x}^{\text {th }}$ pixel value of dimension $\mathrm{K}$ X K.

- Compute the mean of the images $I_{\text {mean }}$ and then subtract it from each image to calculate a matrix say A as follows:

$I_{\text {mean }}=\frac{1}{N} \sum_{n=1}^{N} i_{k}^{n}$ for all $\mathrm{k}=1,2, \ldots \mathrm{K}^{*} \mathrm{~K}$,

Means row wise mean of the above matrix $\mathrm{I}_{\mathrm{N}}$.

$\mathrm{A}=\left[\mathrm{I}_{\mathrm{N}}-\mathrm{I}_{\text {mean }}\right]_{\mathrm{KK}} * \mathrm{~N}$

- Now compute the covariance matrix, $\mathrm{C}_{\mathrm{A}}$ as:

$\mathrm{C}_{\mathrm{A}}=\mathrm{A}^{*} \mathrm{~A}^{\mathrm{T}}$ of dimension $\mathrm{KK} * \mathrm{KK}$.

- Calculate the eigenvectors $\left\{\Omega_{1}, \Omega_{2}, \ldots, \Omega_{N}\right\}$ and eigenvalues $\left\{\lambda_{1}, \lambda_{2}, \ldots, \lambda_{N}\right\}$ of the covariance matrix and arrange the eigenvectors in descending order of their eigenvalues and take the first $\mathrm{M}$ eigenvectors, $\mathrm{M}<\mathrm{K}^{*} \mathrm{~K}$ that correspond to the $\mathrm{M}$ largest eigenvalue. 
- Construct the projection matrix (space of the finger images) W from the selected $\mathrm{M}$ eigenvectors and transform the original dataset which is the reduced dimension of $M$ of the original data of dimension $\mathrm{K}^{*} \mathrm{~K}$.

The eigen fingerprint is given by:

$$
f_{i}=\left[I_{N}\right] * \Omega_{i} \text { where } \Omega_{i} \text { is the eigenvector and } f_{i} \text { are the eigen-fingerprint. }
$$

\subsection{Discrete Wavelet Transform (DWT)}

The discrete wavelet transforms transform the time domain signal to a discrete wavelet representation. In case of fingerprint images DWT segmented multi resolution representation. A two level decomposition is applied to fingerprint image, one along with row and the second one along, which results in four distinct bands LL, LH, HL, and HH where L is low pass filter and $\mathrm{H}$ is high pass filter. Most of the fingerprint information is concentrated in the LL band; the vertical information of the fingerprint image is obtained by LH band, the horizontal information of the fingerprint image is obtained by HL band and the diagonal details of the fingerprint image is obtained $\mathrm{HH}$ band.

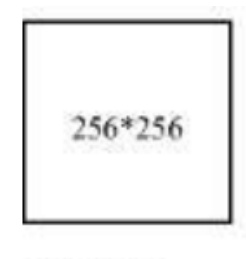

Image to be decomposed

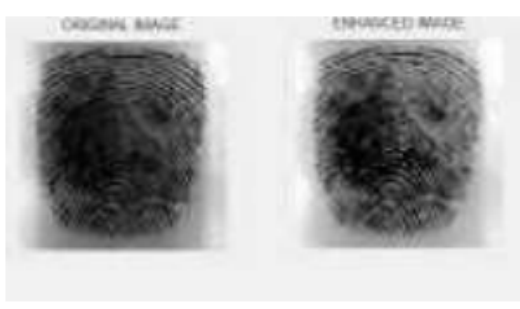

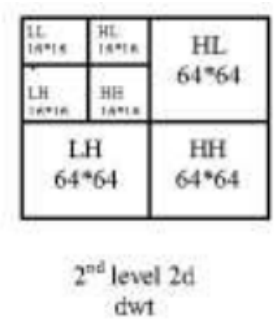

(a)

(b)

Figure 2 (a) DWT sub window image with low and high band filter (b) DWT of fingerprint image

The two dimensional DWT of an image signal requires the 2D filters during the multiplication of separable functions and scaling in $\mathrm{n}_{1}$ (horizontal), $\mathrm{n}_{2}$ (vertical) direction as:

$$
\begin{aligned}
& \varphi\left(n_{1}, n_{2}\right)=\varphi\left(n_{1}\right) \varphi\left(n_{2}\right) \\
& \psi^{H}\left(n_{1}, n_{2}\right)=\psi\left(n_{1}\right) \psi\left(n_{2}\right) \\
& \psi^{V}\left(n_{1}, n_{2}\right)=\psi\left(n_{1}\right) \psi\left(n_{2}\right) \\
& \psi^{D}\left(n_{1}, n_{2}\right)=\psi\left(n_{1}\right) \psi\left(n_{2}\right)
\end{aligned}
$$

Where $\varphi\left(n_{1}, n_{2}\right), \psi^{H}\left(n_{1}, n_{2}\right), \psi^{V}\left(n_{1}, n_{2}\right), \psi^{D}\left(n_{1}, n_{2}\right)$ represents the signal with horizontal, vertical and diagonal details respectively. And the 2D DWT of an fingerprint image function of size $\mathrm{MXN}$ is given as:

$$
\begin{aligned}
& W_{\varphi}\left(j_{0}, k_{1}, k_{2}\right)=\frac{1}{\sqrt{M N}} \sum_{m=0}^{M-1} \sum_{n=0}^{N-1} s(m, n) \varphi_{j_{0}, k_{1}, k_{2}}(m, n) . \\
& W_{\psi}^{i}\left(j_{0}, k_{1}, k_{2}\right)=\frac{1}{\sqrt{M N}} \sum_{m=0}^{M-1} \sum_{n=0}^{N-1} s(m, n) \psi_{j_{0}, k_{1}, k_{2}}^{i}(m, n) .
\end{aligned}
$$

where $\mathrm{i}=\{\mathrm{H}, \mathrm{V}, \mathrm{D}\}$ indicate the directional index of the wavelet function. 


\subsection{Discrete Cosine Transform (DCT)}

Discrete Cosine Transform is a method that transforms an image in spatial domain to frequency domain by partitioning image pixel matrix in blocks of $\mathrm{N} X \mathrm{~N}$ size.

- DCT convert an image into its equivalent frequency

- domain by partitioning image pixel matrix into blocks of size

- $\mathrm{N}^{*} \mathrm{~N}, \mathrm{~N}$ depends upon the type of image.

- DCT convert an image into its equivalent frequency

- domain by partitioning image pixel matrix into blocks of size

- $\mathrm{N}^{*} \mathrm{~N}, \mathrm{~N}$ depends upon the type of image.

In frequency domain, the image is decomposed into many frequency components with most of the image information being concentrated in a part of those components. The energy compaction property along with the removal of redundancy are the main reasons, why it is used for images in the frequency domain. The DCT is one of the most popular frequency transformations in image. A 2D DCT for an image I of size $\mathrm{N} \mathrm{X} \mathrm{N}$ is defined as:

$I\left(k_{1}, k_{2}\right)=\frac{2}{N} u\left(k_{1}\right) u\left(k_{2}\right) \sum_{n 1=0}^{N-1} \sum_{n 2=0}^{N-1} i\left(n_{1}, n_{2}\right) \cos \frac{\pi\left(2 n_{1}+1\right) k_{1}}{2 N} \cos \frac{\pi\left(2 n_{2}+1\right) k_{2}}{2 N} .$.

Where $\mathrm{k}_{1}, \mathrm{k}_{2}=0,1,2, \ldots \mathrm{N}-1$,

$\mu(0)=\frac{1}{\sqrt{2}}$ and $\mu(\mathrm{k})=1$ for $\mathrm{n} \neq 0$

Hence the 2D DCT can be expressed as:

$i\left(k_{1}, k_{2}\right)=\frac{2}{N} \sum_{n 1=0}^{N-1} \sum_{n 2=0}^{N-1} u\left(k_{1}\right) u\left(k_{2}\right) i\left(n_{1}, n_{2}\right) \cos \frac{\pi\left(2 n_{1}+1\right) k_{1}}{2 N} \cos \frac{\pi\left(2 n_{2}+1\right) k_{2}}{2 N} \ldots$

The basic operation of the DCT is as follows for an image of size N X N:

Let $i(k, l)$ is the intensity of the pixel in $\mathrm{k}^{\text {th }}$ row and $\mathrm{l}^{\text {th }}$ column.

$f(u, v)$ is the DCT coefficient in $\mathrm{k}_{1}{ }^{\text {th }}$ row and $\mathrm{k}_{2}^{\text {th }}$ column of the DCT matrix.

Since much of signal energy in most of images is at low frequencies, those appear in upper left corner of DCT.

And since higher frequencies lies at lower right corner, Feature compression is achieved and are often small.

Basically an $8 \mathrm{X} 8$ array is a input to the DCT which contains each pixel's gray scale value of 8 bit from 0 to 255 .

\subsection{Local Binary Pattern (LBP)}

The LBP is a feature extraction method, which labels the pixels of an image to binary number by thresholding the neighbourhood of each pixel. Due to its discriminative power and computational simplicity, LBP is used for texture classification in 2D shaped image. The basic idea of LBP is that the image is subdivided into blocks of size $3 \mathrm{X} 3$, then in each block the central pixel value is used as a threshold for its 8 neighbour pixels to binarize the code and the equation of constructing LBP is given as:

$$
\operatorname{LBP}\left(i_{x}, i_{y}\right)=\sum_{i=0}^{7} 2^{n} h\left(I_{n}-I\left(i_{x}, i_{y}\right)\right)
$$


Where $\operatorname{LBP}(i x, i y)$ is the LBP value at centre pixel, Inand $I(i x, i y)$ are the values of neighbour pixel and centre pixel respectively and convert to the gray values of 8 surrounding pixels by function $\mathrm{s}$ is defined as:

$$
s(x)=\{1 \text { if } x \geq 0 \text { and } 0 \text { if } x<0
$$

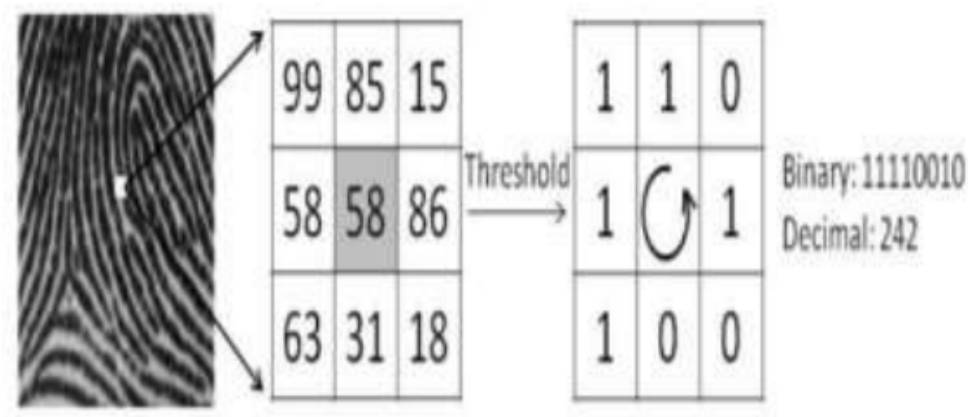

Figure 3 LBP 3 X3 sub window for binarization

\subsection{Minutiae Feature Extraction}

The major features of a fingerprint image are minutiae points. In this study it was observed that most work done on two minutiae points 1) ridge ending and 2) ridge bifurcation. A ridge ending is a point that ends abruptly and a bifurcation is a point where it diverges into branches. It also has been said that a quality fingerprint image can have 25-80 minutiae points as per its resolution. Basically a minutiae $\mathrm{m}_{\mathrm{i}}$ can be described by four parameters as:

$m_{i}=\left(x_{i}, y_{i}, \theta_{i}, t_{i}\right)$, Where $\mathrm{x}_{\mathrm{i}}$ and $\mathrm{y}_{\mathrm{i}}$ are coordinates of the minutiae point, $\theta_{\mathrm{i}}$ is the direction from local ridge orientation and $t_{i}$ is the type of minutiae points ( ridge ending, bifurcation etc)

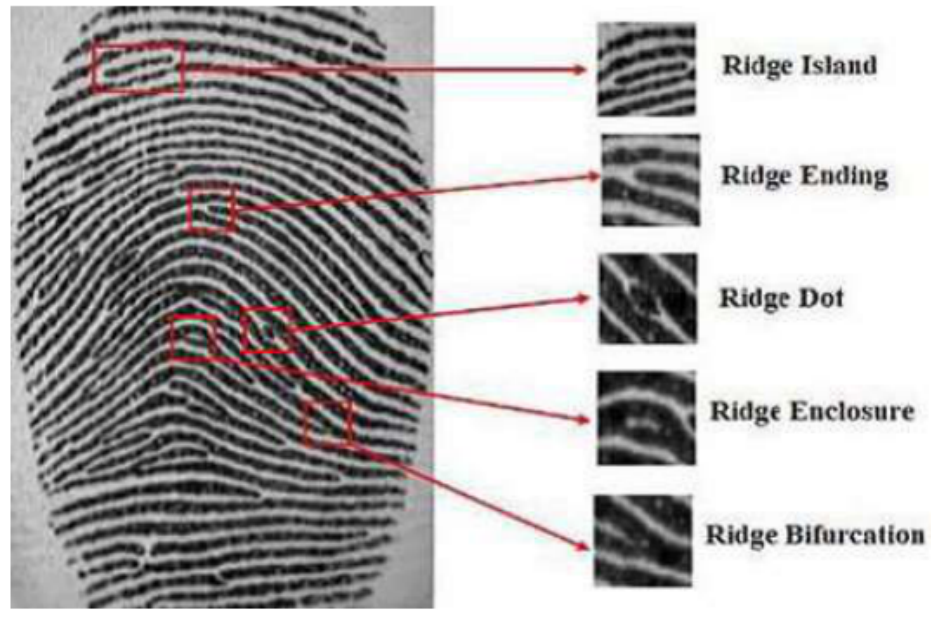

Figure 4 Minutiae features of a fingerprint Image

To find out minutiae points of a fingerprint image, it first goes through binarization and ridge thinning process. Then minutiae are calculated using a $3 \mathrm{X} 3$ window size of image by considering local neighbourhood of each pixel of that window. Most of work done for minutiae extraction was through crossing number $(\mathrm{CN})$. The $\mathrm{CN}$ value is computed which is described as follows: 
Let a $3 \mathrm{X} 3$ block of an image with its neighbourhood of each pixel is shown as follows:

\begin{tabular}{|c|c|c|}
\hline$P_{4}$ & $P_{3}$ & $P_{2}$ \\
\hline$P_{5}$ & $P$ & $P_{1}$ \\
\hline$P_{6}$ & $P_{7}$ & $P_{8}$ \\
\hline
\end{tabular}

Figure 5: A $3 X 3$ block image for minutiae Crossing Number

Then the crossing number is computed as:

$$
C N_{(x, y)}=\frac{1}{2} \sum_{i=1}^{8}\left|p_{i}-p_{i+1}\right|, \quad p_{1}=p_{9}
$$

It is defined as half the sum of the differences between adjacent pixel pairs in the eightneighbourhood. From the properties of the crossing number one can classify a ridge pixel as an ending, bifurcation or non-minutiae point as shown in following table.

Table 1: Properties of Crossing Number

\begin{tabular}{|c|l|}
\hline CN & \multicolumn{1}{|c|}{ PROPERTY } \\
\hline 0 & Isolated Point \\
\hline 1 & Ridge Ending Point \\
\hline 2 & Continuing ridge Point \\
\hline 3 & Bifurcation Point \\
\hline 4 & Crossing Point \\
\hline
\end{tabular}

\section{CONCLUSIONS}

The main objective of our survey is to introduce various feature extraction methods used for fingerprint image pattern to readers and researchers. Basically these feature extraction methods are used to reduce the dimensionality of the original image from a larger feature dataset to a smaller feature dataset. It has also been observed that the feature extraction methods were chosen to keep the most valuable features and discard the least reliable features of image for better identification, recognition and classification.

Many feature extraction techniques have been developed so far are discussed here in our survey. In this survey, we also discussed which feature extraction method will be better for the real world dataset, depending upon the requirement and application to be used. Here we present an overview of the recent feature extraction algorithms proposed so far in literature. This study also discuss the steps of various feature extraction methods by which one can choose the appropriate method for the further phases of fingerprint image identification, recognition and classification.

\section{REFERENCES}

[1] Moayer, B. and K.S. Fu, "A tree system approach for fingerprint pattern recognition", IEEE Trans. Pattern Analysis and Machine Intelligence, 8(3):376-388, 1986.

[2] Verma, M.R., A.K. Majumdar, and B. Chatterjee, "Edge detection in fingerprints, PatternRecognition”, 20(5):513-523, 1987.

[3] O'Gorman, L. and J. Nickerson, “An approach to fingerprint filter design, Pattern Recognition", 22:29-38, 1989. 
[4] Sherlock, B.G., D.M. Monro, and K. Millard, "Fingerprint enhancement by directional Fourier filtering”, IEE Proc. Vis. Image Signal Processing, 141:87-94, 1994.

[5] Szekely, E.N. and V. Szekely, "Image recognition problems of fingerprint identification", Microprocessor and Microsystems, 17(4):215, 1993.

[6] [6]. Mehtre, B.M., "Fingerprint image analysis for automatic identification", Machine Vision and Applications, 6(2-3):124-139, 1993.

[7] Weber, D.M., "A cost effect verification algorithm for commercial application", Proc. 1992 South African Symposium on Communications and Signal Processing, pp. 99-104, 1992.

[8] Leung, M.T., W.E. Engeler, and P. Frank, "Fingerprint image processing using neural network", Proc. Tenth Conf. Computer and Communication Systems, pp. 582-586, 1990.

[9] Leung, W.F., S.H. Leung, W.H. Lau, and A. Luk, "Fingerprint recognition using neural Network", Proc. IEEEWorkshop Neural Network for Signal Processing, pp. 226-235, 1991.

[10] Hung. D.C.D. "Enhancement and feature purification of fingerprint image", Pattern Recognition, 26:1661-1671, 1993.

[11] Xiao, Q. and H. Raafat," Fingerprint image postprocessing: A combined statistical and structural approach", Pattern Recognition, 24(10):985-992, 1991.

[12] Jianjiang Feng and Anil K. Jain , "FM model based fingerprint reconstruction from Minutiae template", Department of Computer Science and Engineering, Michigan State University.

[13] Arun Ross, Sarat C.Dass, Anil K.Jain , "Estimating Fingerprint Deformation", International Conference on Biometric Authentication (ICBA),LNCS vol. 3072, pp. 249255, Hong Kong, July 2004.

[14] Yi Chen, Anil K. Jain , "Dots and incipients: extended features for partial fingerprint Matching", Michigan state university.

[15] M. Tico, E. Immonen, P. Ramo, P. Kuosmanen, and J, Saarinen, " Fingerprint Recognition Using Wavelet Features", The 2001 IEEE International Symposium on circuit and systems, Vol.2. pp. 21-24, 2001

[16] S. Tachaphetpiboon and T. Amornraksa, "A fingerprint matching method using DCT features", Proceedings of ISCIT 2005, pp.446-449.

[17] S.Malathi and Dr.C.Meena," An efficient method for partial fingerprint recognition based on Local Binary Pattern" International Conference on Communication, Control and Computing Technologies, 2010, pp. 569-572.

[18] M.P Dale, M.A Joshi, M.K Sahu, “ DCT Feature Based Fingerprint Recognition”, International Conference on Intelligent and advanced systems, 2007, pp. 611-615.

[19] C. J. Lee and S. D. Wang, "Fingerprint Feature Extraction Using Gabor Filters," Electronics Letters, Vol. 35, No. 4, 1999, pp. 288-290. doi:10.1049/el:19990213

[20] Nanni, Loris \& Lumini, Alessandra. "Local binary patterns for a hybrid fingerprint matcher". Pattern Recognition, ELSEVIER, 41. 3461-3466. 10.1016/j.patcog.2008.05.013.

[21] Shashi Kumar D R, K B Raja, R K Chhotaray, and Sabyasachi Pattanaik, "DWT Based Fingerprint Recognition using Non Minutiae Features", IJCSI, International Journal of Computer Science Issues, Vol. 8, Issue 2, March 2011, pp. 257-265. 
[22] Yongxu, Wang \& Xinyu, Ao \& Yuanfeng, Du \& Li, Yongping, "A Fingerprint Recognition Algorithm Based on Principal Component Analysis". TENCON 2006 - 2006 IEEE Region 10 Conference 1 - 4. 10.1109/TENCON.2006.344032.

[23] Chhaya Sunil Khandelwal a , Ranjan Maheshwarib , U.B.Shinde," Review paper on applications of principal component analysis in multimodal biometrics system", 2nd International Conference on Intelligent Computing, Communication \& Convergence (ICCC-2016), ELSEVIER, Procedia Computer Science 92 ( 2016 ), pp.481 - 486.

[24] Hong, L.,Y.Wan, andA. Jain, "Fingerprint image enhancement: Algorithm and performance evaluation", IEEE Trans. Pattern Analysis and Machine Intelligence, 20:777-789, 1998.

[25] Jain, A. and F. Farrokhnia, "Unsupervised texture segmentation using Gabor filters", Pattern Recognition, 24:1167-1186, 1991.

[26] Soille, P., "Morphological Image Analysis: Principles and Applications", New York: Springer-Verlag, 1999, pp. 173-174.

[27] Coetzee, L, and E.C. Botha, "Fingerprint recognition in low quality images", Pattern Recognition, 26:1441-1460, 1993.

[28] Ratha, N.K., S. Chen, A. Jain, "Adaptive flow orientation-based feature extraction in fingerprint images", Pattern Recognition, 28:1657-1672.

[29] Canny, J. “A computational approach to edge detection", IEEE Trans. Pattern Analysis and Machine Intelligence, 8:679-698, 1986.

[30] Marr, D. and E. Hildreth, “Theory of edge detection”, Proc. Royal Society of London B, 207:187-217, 1980.

[31] Perona, P., "Steerable-scalable kernels for edge detection and junction analysis, Image Vision Comput"., 10:663-672, 1992.

[32] Maio, D. and D. Maltoni, "Direct gray-scale minutia detection in fingerprints", IEEE Trans. Pattern Analysis and Machine Intelligence, 19:27-40, 1997.

[33] Roddy, A.R. and J.D. Stosz, "Fingerprint features-statistical analysis and system performance estimates", Proc. IEEE, 85:1390-1421, 1997.

[34] Otsu, N.,"Athreshold selection method from gray-level histogram”, IEEE Trans. Sys., Man, and Cybernetics, 9:62-66, 1979.

[35] D. Maltoni, D. Maio, A. K.Jain, and S. Prabhakar, "Handbook of Fingerprint Recognition", Springer- Verlag, New York, 2003.

[36] T. Nakamura, H. Fujiwara, M. Hirooka, and K. Sumi, "Fingerprint Enhancement Using a Parallel Ridge Filter", 17th International Conference on Pattern Recognition 2004, Cambridge, United Kingdom,2004.

[37] Yager N, Amin A “Fingerprint classification: a review”. Pattern Anal Appl 7:77-93

[38] Maltoni D, Maio D, Jain AK, Prabhakar S, "Handbook of fingerprint recognition". Springer, Berlin

[39] Candela G, "Pcasys-a pattern-level classification automation system for fingerprints". NIST technical report NISTIR 5647

[40] Chong M, Ngee T, Jun L, Gay K, "Geometric framework for fingerprint image classification”. Pattern Recognit 30(7-9):1475-1488 